\title{
Anomalous Resonance Phenomena of Solitary Waves with Internal Modes
}

\author{
Niurka R. Quintero* and Angel Sánchez ${ }^{\dagger}$ \\ Grupo Interdisciplinar de Sistemas Complicados (GISC), Departamento de Matemáticas, Universidad Carlos III de Madrid, \\ Avenida de la Universidad 30, E-28911 Leganés, Madrid, Spain \\ Franz G. Mertens \\ Physikalisches Institut, Universität Bayreuth, D-95440 Bayreuth, Germany
}

(Received 16 July 1999)

\begin{abstract}
We investigate the nonparametric, pure ac driven dynamics of nonlinear Klein-Gordon solitary waves having an internal mode of frequency $\Omega_{i}$. We show that the strongest resonance arises when the driving frequency $\delta=\Omega_{i} / 2$, whereas when $\delta=\Omega_{i}$ the resonance is weaker, disappearing for nonzero damping. At resonance, the dynamics of the kink center of mass becomes chaotic. As we identify the resonance mechanism as an indirect coupling to the internal mode due to its symmetry, we expect similar results for other systems.
\end{abstract}

PACS numbers: $05.45 . \mathrm{Yv}, 02.30 . J r, 03.50 .-\mathrm{z}, 63.20 . \mathrm{Pw}$

An important paradigm established over the last two decades is that solitary waves or solitons behave very much like point particles when subjected to (a large class of) external forces and perturbations [1-3]. However, many solitary waves possess one (sometimes more than one) internal or shape mode [4,5], and in that case the particle picture of their dynamics may be oversimplified: Indeed, the internal mode can temporarily store energy and release it at a later stage, giving rise to resonance phenomena in solitary wave collisions [5] or in solitary wave interactions with inhomogeneities [6]. As internal modes are quite common in nonlinear systems, either intrinsically or as a result of small perturbations [7], the question of their influence on the dynamics of solitary waves is a very general and relevant one.

One aspect of solitary wave dynamics that has proven itself difficult to understand is that of topological solitary waves or kinks subjected to pure, i.e., nonparametric ac driving. Thus, only recently [8] the ac driven dynamics of sine-Gordon kinks (that do not possess an internal mode) has been definitely clarified. Naively, the only new phenomenon one expects when a nonparametric external driving acts on solitons with internal modes is a resonance when its frequency, $\Delta$, matches that of an internal mode, $\Omega_{i}$. The aim of this Letter is to show that, in fact, the actual scenario is most unexpected and highly nontrivial. As we will see below, a strong, anomalous resonance arises when $\delta=\Omega_{i} / 2$, whereas the normal resonance at $\delta=\Omega_{i}$ is definitely weaker, only possible at exactly zero damping, and even then it can be suppressed by appropriate choices of other parameters. We expect this result to be generic, because our analytical approach allows us to identify the mechanism for such a peculiar phenomenon: The ac force does not act directly on the internal mode (because of symmetry reasons), but rather, they interact indirectly via the translational motion which couples to the internal mode. Our predictions are fully confirmed by numerical simu- lations, which in addition show the implications of these resonances for the kink dynamics.

As a specific example of a kink with internal mode, we take the well known [1] $\phi^{4}$ equation, which, when driven with an ac force $f(t)=\epsilon \sin \left(\delta t+\delta_{0}\right)$, reads

$$
\phi_{t t}-\phi_{x x}+U^{\prime}(\phi)=-\beta \phi_{t}+f(t),
$$

where $U(\phi)=\left(\phi^{2}-1\right)^{2} / 4$ and $\beta$ is a damping coefficient. Previous related works on this system are [9], where resonances in the presence of an external (time independent) potential have been considered, and [10], which dealt with nonresonant, high frequency parametric ac drivings. For our problem, our analytical approach will be the well known collective coordinate (CC) method [2,3]. A first order of approximation is given by the McLaughlin-Scott method [11]: We assume that the solution of (1) is of the form

$$
\phi(x, t)=\tanh \left[\frac{x-X(t)}{l_{0} \sqrt{1-V(t)^{2}}}\right],
$$

where $l_{0}=\sqrt{2}$. The center of the kink $X(t)$ and its velocity $V(t)$ are related by $X(t)=\int_{0}^{t} d t^{\prime} V\left(t^{\prime}\right)+X(0)$, and both are unknown functions describing the motion of the kink as a coherent entity. By means of a standard procedure $[2,3,8,11]$ involving conservation laws, an ordinary differential equation of motion for $V(t)$ can be obtained, linearized [8], and solved, yielding

$$
\begin{gathered}
V(t)=\frac{r(t)}{\sqrt{1+r(t)^{2}}}, \\
r(t) \equiv c e^{-\beta t}-\frac{3 \sqrt{2} \epsilon}{2\left(\beta^{2}+\delta^{2}\right)} \\
\times\left[\beta \sin \left(\delta t+\delta_{0}\right)-\delta \cos \left(\delta t+\delta_{0}\right)\right], \\
c=\gamma_{0} V(0)+\frac{3 \sqrt{2} \epsilon}{2\left(\beta^{2}+\delta^{2}\right)}\left[\beta \sin \left(\delta_{0}\right)-\delta \cos \left(\delta_{0}\right)\right],
\end{gathered}
$$

2000 The American Physical Society 
where $\gamma_{0} \equiv 1 / \sqrt{1-V(0)^{2}}$. For the undamped case $(\beta=0)$, we see that the kink oscillates if $\gamma_{0} V(0)=$ $3 \sqrt{2} \epsilon \cos \left(\delta_{0}\right) /(2 \delta)$; otherwise, the kink will move either to the right or to the left, depending on the relation between the parameters of the ac force and the initial velocity. This dc motion is absent when $\beta \neq 0$, as has been numerically confirmed for sine-Gordon kinks in [8].

In order to include internal mode effects, we proceed as follows: We rewrite Eq. (1) as

$$
\dot{\psi}=-\frac{\delta H}{\delta \phi}-\beta \dot{\phi}+f(t), \quad \dot{\phi}=\frac{\delta H}{\delta \psi},
$$

where $\psi=\dot{\phi}$, the dot meaning derivative with respect to time, and

$$
H=\int_{-\infty}^{+\infty} d x\left\{\frac{1}{2} \psi^{2}+\frac{1}{2} \phi_{x}^{2}+U(\phi)\right\}
$$

is the Hamiltonian of the system when $\epsilon=\beta=0$. We now make the ansatz $\phi(x, t)=\phi[x-X(t), l(t)]$, whereas from the definition of $\psi$ we have that $\psi(x, t)=$ $\psi[x-X(t), l(t), \dot{X}, i] . \quad$ As in the McLaughlin-Scott method, $X(t)$ represents the kink center position, but now we introduce a second collective variable $l(t)$ that will stand for the kink width, i.e., the internal mode excitation, below.

The procedure to obtain the $\mathrm{CC}$ equations corresponding to this generalized traveling wave ansatz has been put forward in [12,13]. Basically, it consists of inserting our ansatz into (5), multiplying the first equation by $\partial \phi / \partial X$ and the second one by $\partial \psi / \partial X(\partial \phi / \partial l$ and $\partial \psi / \partial l)$, taking their difference, and integrating over $x$. This yields a rather cumbersome, ordinary differential equation for $X(t)$ $[l(t)]$, which we omit here for brevity. The next step is to choose a specific functional form for $\phi$, which we do following the work of Rice [14], and let

$$
\phi[x-X(t), l(t)]=\phi_{0}\left[\frac{x-X(t)}{l(t)}\right],
$$

where $\phi_{0}\left[(x-X) / l_{0}\right]$ is the static kink solution, which is an odd function (with respect to its center) for the $\phi^{4}$ and for any other even potential. Upon particularization of the CC equations for this form for $\phi$ we finally obtain

$$
\begin{gathered}
M_{0} l_{0} \frac{\ddot{X}}{l}-M_{0} l_{0} \frac{\dot{X} \dot{l}}{l^{2}}=-\beta M_{0} l_{0} \frac{\dot{X}}{l}-2 f(t), \\
\alpha M_{0} l_{0} \frac{\ddot{l}}{l}+M_{0} l_{0} \frac{\dot{X}^{2}}{l^{2}}=K^{i n t}(l, \dot{l}, \dot{X})-\beta \alpha M_{0} l_{0} \frac{\dot{l}}{l} ;
\end{gathered}
$$

where $K^{i n t}=-\partial E / \partial l$ and

$$
E=\frac{1}{2} \frac{l_{0}}{l} M_{0} \dot{X}^{2}+\frac{l_{0}}{2 l} \alpha M_{0} \dot{l}^{2}+\frac{1}{2} M_{0}\left(\frac{l_{0}}{l}+\frac{l}{l_{0}}\right)
$$

is the kink energy. Importantly, in obtaining Eq. (9) a term of the form $f(t) \int_{-\infty}^{\infty} d x \partial \phi_{0} / \partial l$, coming from the coupling of the ac driving to the internal mode, has vanished because of symmetry. For the $\phi^{4}$ equation, $\phi_{0}=\tanh x$ in Eq. (7), which yields $\alpha=\left(\pi^{2}-6\right) / 12$ and $M_{0}=2 \sqrt{2} / 3$.

Let us now simplify these expressions in order to make its physical significance more transparent. To begin with, Eq. (8) can be solved for $\dot{X} / l$, yielding

$$
\begin{aligned}
P \equiv & \frac{M_{0} l_{0} \dot{X}}{l} \\
= & -2 \epsilon \frac{\beta \sin \left(\delta t+\delta_{0}\right)-\delta \cos \left(\delta t+\delta_{0}\right)}{\left(\beta^{2}+\delta^{2}\right)} \\
& +e^{-\beta t}\left[2 \epsilon \frac{\beta \sin \left(\delta_{0}\right)-\delta \cos \left(\delta_{0}\right)}{\left(\beta^{2}+\delta^{2}\right)}+\frac{M_{0} l_{0} V(0)}{l_{s}}\right],
\end{aligned}
$$

where $l_{s}=l_{0} / \gamma_{0}$. Inserting Eq. (11) into Eq. (9), we find

$$
\alpha\left[\dot{l}^{2}-2 \ddot{l}-2 \beta \dot{l i}\right]=\frac{l^{2}}{l_{0}^{2}}\left[1+\frac{P^{2}}{M_{0}^{2}}\right]-1 .
$$

Although Eqs. (11) and (12) are quite complicated, choosing $V(0)$ and the phase $\delta_{0}$ so that the exponential terms in Eq. (11) vanish, we can use a change of variables (proposed in [15] for the dc driving case) to transform the Eq. (12) into a Pinney-like equation (see [16] and references therein), which can be solved in terms of Mathieu functions [17] if $\beta=0$ (when $\beta \neq 0$ we have not been able to solve this problem analytically).

In any event, we do not need the analytical expressions for the solution of the system (11) and (12) to understand the physics predicted by our approach. The term $P^{2}$ in Eq. (12) is an oscillatory function with frequency $2 \delta$ [see Eq. (11)]; hence, we can immediately expect a resonance when the external frequency $\delta$ is half the frequency of the internal mode, $\Omega_{R}=1 / \sqrt{\alpha} l_{0}$ in the Rice approximation. For the $\phi^{4}$ model $\Omega_{R}$ overestimates $\Omega_{i}=\sqrt{3 / 2}$ by $1.7 \%$ [14]. The analytical solution for $\beta=0$ confirms this expectation, while numerical integration of Eq. (12) proves that the behavior of $l$ is that of a resonant, damped oscillator when $\beta \neq 0$. When $\delta=\Omega_{R}$, inspection of Eqs. (11) and (12) leads to the conclusion that another resonance should be found at $\delta=\Omega_{R}$ only if $\beta=0$ (otherwise it is a transient phenomenon of lifetime $\beta^{-1}$ ); even then, by choosing $V(0)$ and $\delta_{0}$ to cancel the nonoscillatory terms in Eq. (11) the resonance is completely suppressed. Furthermore, both analytically and numerically we have verified that, far away from the resonances, the behavior of the kink center, $X(t)$, is practically the same as the one predicted by the McLaughlin-Scott approach, Eq. (3). This means that, within the CC framework, the behavior of ac driven $\phi^{4}$ kinks is described by the McLaughlin-Scott ansatz, and only for drivings close to $\Omega_{R} / 2$ (and $\Omega_{R}$ if $\beta=0$ ) such approach fails and resonant phenomena arise.

At this point, two key issues must be addressed: First, underlying $\mathrm{CC}$ methods is the assumption that no (or a negligible amount of) radiation is generated by the perturbation, an assumption whose validity can be assessed only through comparison with the corresponding PDE. Second, even if that is the case, the CC equations predict 
an unbounded growth of $l(t)$ at resonance, and it is difficult to understand what that means in physical terms for the kink of the full PDE, whose width is controlled by the properties of the equation. In view of this, we have computed the numerical solution of the PDE (1) by using the conservative Strauss-Vázquez scheme [18] with $\Delta x=0.1, \Delta t=0.01$, and a total system length of $L=400$. Our initial condition was a kink at rest and $\delta_{0}=\pi / 2$, a pair of values for which we should not see a resonance at $\Omega_{i}$. We come back to this below. We monitored the position and the velocity of the kink center as well as the total energy in the system, computed from the Hamiltonian (6). We also tried to measure directly the kink width, but we found that it is quite complicated to estimate it from the numerics, this being the reason why we have resorted to less direct measurements.

Figure 1 shows examples of the kink center dynamics and its energy evolution both close to and away from the predicted resonance. Off-resonance, the behavior of both magnitudes is periodic, whereas at the resonance it becomes chaotic. Specifically, the energy increases with time: The closer to the resonance value, the faster the increment. The center motion is initially periodic, until the internal mode amplitude has increased too much and stored too much energy, subsequently releasing it through its coupling with the translation mode (which we know exists from [5]), eventually yielding the kink motion erratic as this process is repeated once and again. This is a clear evidence in favor of the resonance predicted from the $\mathrm{CC}$ treatment. We have verified that at resonance the kink motion is very sensitive to changes in the initial conditions, hence our claim of the appearance of chaos.

Figure 2 depicts the resonance as seen through the mean energy, computed as a time average from $t=10000$ (after transients have died out in the damped case) to the end of the run at $t=25000$. Figure 2(a) shows the behavior of this magnitude around $\Omega_{i} / 2$. It is clear from the plot that there is a strong resonance at $\delta \approx 0.6102 \approx \Omega_{i} / 2$, both with and without dissipation. In fact, some points are missing in the $\beta=0$ line because the corresponding kinks, in their apparently random motion, left the system before the end of the run. On the other hand, in spite of our choice of initial conditions, for which no resonance is predicted at $\Omega_{i}$ for $\beta=0$, a weak resonance can be seen at $\delta \approx 1.225 \approx \Omega_{i}$ in Fig. 2(b). We note that the other two small peaks are spurious, since they appear, disappear, or change location depending on the choice of the length for the numerical simulation. We believe that this discrepancy might come from the difficulty of numerically tuning the condition for its suppression. In any event, that would be a very special case, and the behavior we find for these parameters is representative of what occurs for other choices of the initial velocity and the phase. Those give rise to a similar behavior, while, remarkably, the resonance at $\Omega_{i}$ is always weaker and narrower than that at $\Omega_{i} / 2$ (cf. Fig. 2). We also see that the prediction that the resonance at $\Omega_{i}$ is suppressed by the dissipation is also confirmed, the small
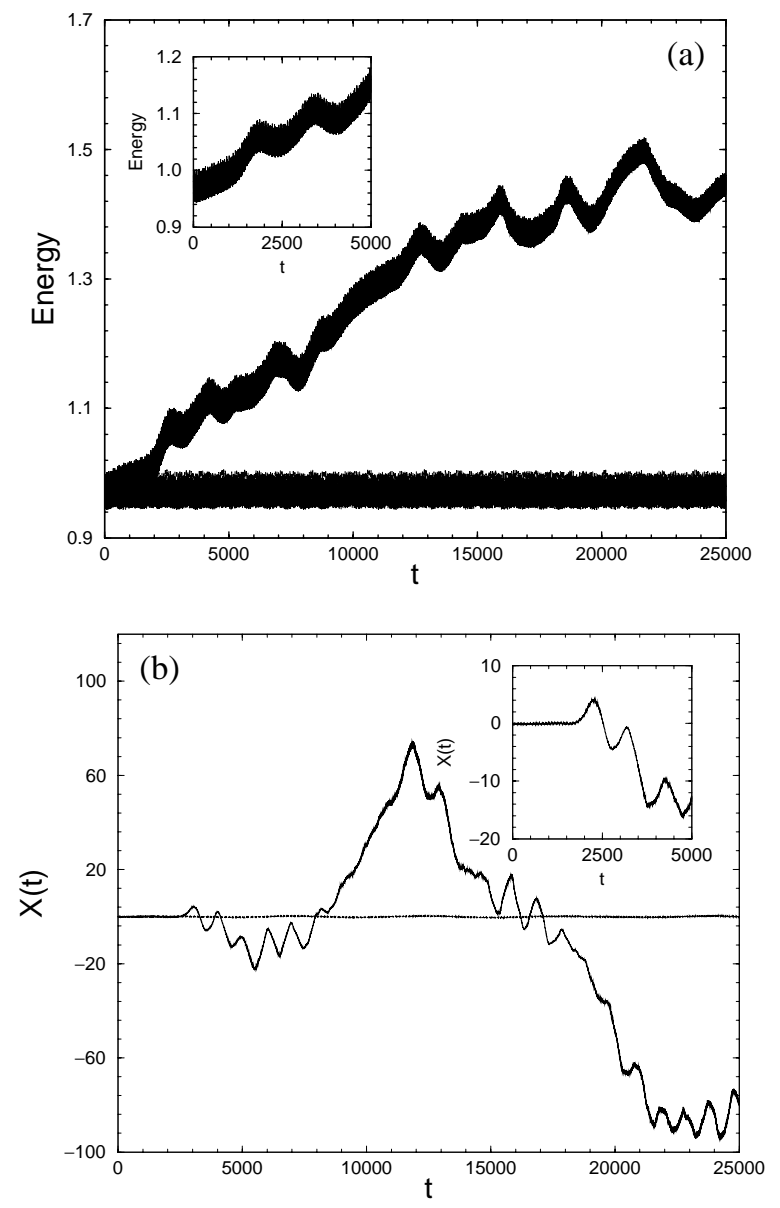

FIG. 1. Results for the PDE (1) with $\beta=0, \epsilon=0.01$. (a) Total energy when $\delta=0.6100$ (upper line), $\delta=0.6080$ (lower line). (b) Same for the kink center, $X(t)$. Insets show the same for $\delta=0.6102$, a value for which the kink reaches the boundary of the numerical system before $t=25000$.

peak in the lower line stemming from the transients, which are not exactly zero for $10000 \leq t \leq 25000$. Another interesting remark is that Fourier analysis shows that the monotonous increasing of the energy that appears in Fig. 2(b) comes from the fact that, when $\delta \gtrsim 1.1$, the lowest phonon mode (with frequency $\omega_{p}=\sqrt{2}$ ) begins to be excited, the amplitude of its excitation monotonically increasing as $\delta \rightarrow \sqrt{2}$. No evidence for lowest phonon mode excitation is seen for the resonance at $\Omega_{i} / 2$; therefore, this is indeed a phenomenon arising from the coupling of the translation and the internal mode as predicted by the $\mathrm{CC}$ calculation.

In summary, we have studied how ac forces affect solitary waves of kink type with an internal mode of frequency $\Omega_{i}$. Specifically, we have clearly shown that the behavior of $\phi^{4}$ kinks under ac driving is very well described by the two-variable CC theory we have developed here. The main feature of the nonparametrically, ac driven $\phi^{4}$ kink dynamics is that the strongest resonance occurs at $\delta=\Omega_{i} / 2$, and not at the frequency one would expect, $\delta=\Omega_{i}$. We emphasize that this novel resonance phenomenon is totally unexpected from the knowledge of the internal mode frequency, and arises 

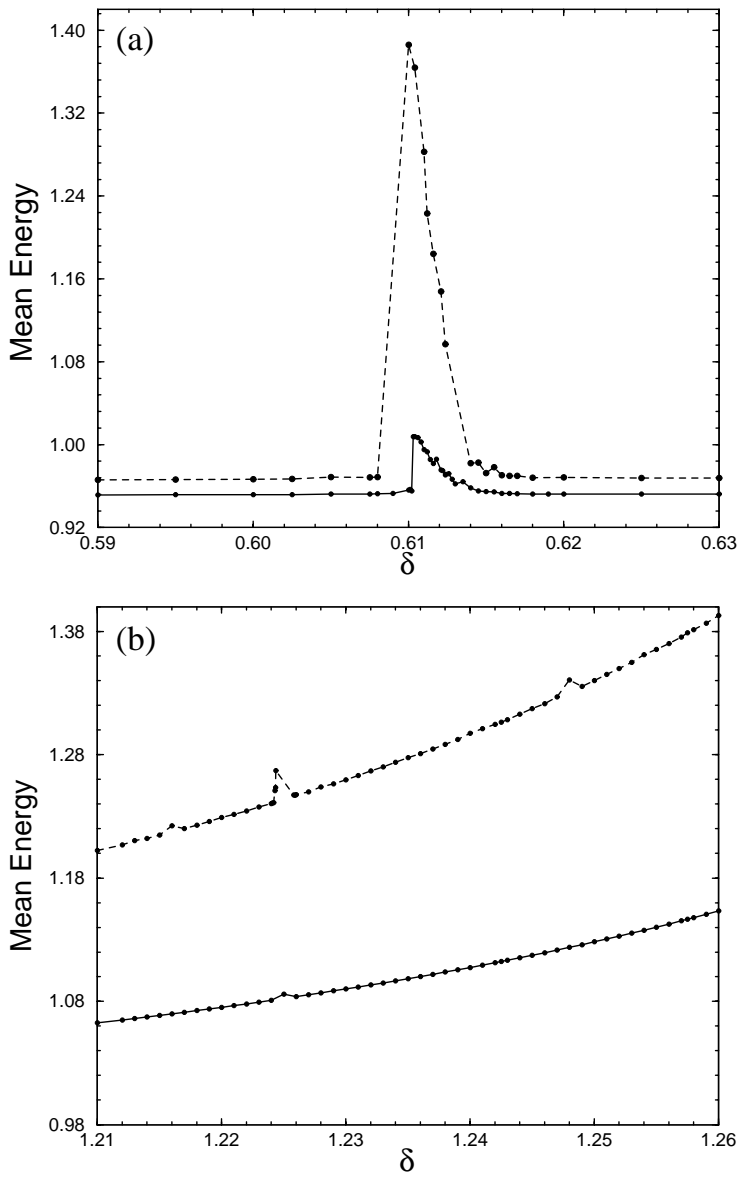

FIG. 2. Results for the PDE (1). Mean value of the energy (see text for the way it is computed) vs driving frequency $\delta$, (a) close to $\Omega_{i} / 2$, (b) close to $\Omega_{i}$. In both cases, the upper line corresponds to $\beta=0$, and the lower line to $\beta=0.001$.

from the indirect interaction of the external force with the internal mode via the translational motion. Although our results have been obtained for a specific example, the $\phi^{4}$ equation (1), other models with even on-site potentials and internal modes, such as the double sine-Gordon equation, for instance, will behave similarly because a CC approach will lead to analogous results. The resonance found at the CC level manifests itself at the PDE level as erratic or chaotic (strongly dependent on the initial conditions) motion of the kink as the kinetic energy of the center of mass is stored into, and recovered from, the internal mode.

To conclude, we note that the anomalous resonances described here are important on their own, as examples of the highly nontrivial behavior of nonlinear systems and as hints about the mechanisms governing kink dynamics. In addition, we think that this phenomenon should be very general, in view of the recent finding [7] that perturbations of kink-bearing nonlinear systems often lead to the development of an internal mode. As this occurs in the discrete sine-Gordon model [7], a resonance like the one discussed here could be relevant for the mode locking phenomena reported for that system in [19]. Finally, by using this discreteness induced internal mode, we point out that the resonance we find could be observed in experiments by using Josephson junction arrays as in [20].

We thank Yuri Gaididei, Francisco Domínguez-Adame, and José Cuesta for discussions. Work at GISC (Leganés) has been supported by DGESIC (Spain) Grant No. PB960119. Travel between Bayreuth and Madrid has been supported by "Acciones Integradas Hispano-Alemanas," a joint program of DAAD (Az. 314-AI) and DGESIC.

*Electronic address: kinter@math.uc3m.es

†Electronic address: anxo@math.uc3m.es

†Electronic address:

franz.mertens@theo.phy.uni-bayreuth.de

[1] A. C. Scott, Nonlinear Science (Oxford University, Oxford, 1999).

[2] A. Sánchez and A. R. Bishop, SIAM Rev. 40, 579 (1998), and references therein.

[3] Yu. S. Kivshar and B. A. Malomed, Rev. Mod. Phys. 61, 763 (1989), and references therein.

[4] M. Peyrard and M. Remoissenet, Phys. Rev. B 26, 2886 (1982).

[5] M. Peyrard and D. K. Campbell, Physica (Amsterdam) 9D, 33 (1983); D. K. Campbell, J. F. Schonfeld, and C. A. Wingate, ibid. 9, 1 (1983); D. K. Campbell, M. Peyrard, and P. Sodano, ibid. 19, 165 (1986).

[6] Yu. S. Kivshar, F. Zhang, and L. Vázquez, Phys. Rev. Lett. 67, 1177 (1991); F. Zhang, Yu. S. Kivshar, and L. Vázquez, Phys. Rev. A 46, 5214 (1992).

[7] Yu. S. Kivshar, D.E. Pelinovsky, T. Cretegny, and M. Peyrard, Phys. Rev. Lett. 80, 5032 (1998).

[8] N. R. Quintero and A. Sánchez, Phys. Lett. A 247, 161 (1998); Eur. Phys. J. B 6, 133 (1998).

[9] J. A. González, B. A. Mello, L. I. Reyes, and L. E. Guerrero, Phys. Rev. Lett. 80, 1361 (1998).

[10] Yu. S. Kivshar and A. Sánchez, Phys. Rev. Lett. 77, 582 (1996).

[11] D. W. McLaughlin and A.C. Scott, Phys. Rev. A 18, 1652 (1978).

[12] G. M. Wysin, F. G. Mertens, A. R. Völkel, and A. R. Bishop, in Nonlinear Coherent Structures in Physics and Biology, edited by K.H. Spatschek and F. G. Mertens, NATO ASI, Ser. B, Vol. 329 (Plenum, New York, 1994).

[13] F. G. Mertens, H. J. Schnitzer, and A. R. Bishop, Phys. Rev. B 56, 2510 (1997).

[14] M. J. Rice, Phys. Rev. B 28, 3587 (1983).

[15] E. Majerníková, Yu. B. Gaididei, and O. M. Braun, Phys. Rev. E 52, 1241 (1995).

[16] J. L. Reid and J. R. Ray, Z. Angew. Math. Mech. 64, 365 (1984).

[17] N. R. Quintero, A. Sánchez, and F. G. Mertens (unpublished).

[18] W. A. Strauss and L. Vázquez, J. Comput. Phys. 28, 271 (1978).

[19] P. J. Martínez, F. Falo, J. J. Mazo, L. M. Floría, and A. Sánchez, Phys. Rev. B 56, 87 (1997).

[20] F. Falo, P. J. Martínez, J. J. Mazo, and S. Cilla, Europhys. Lett. 45, 700 (1999). 